University of Wollongong

Research Online

Faculty of Engineering and Information

Faculty of Engineering and Information

Sciences - Papers: Part A

Sciences

$1-1-2016$

\title{
Bottom-up microfabrication process for individually controlled conjugated polymer actuators
}

Alexandre Khaldi

Linkoping University, ak794@cam.ac.uk

Ali Maziz

Universite de Cergy-Pontoise

Gursel Alici

University of Wollongong, gursel@uow.edu.au

Geoffrey M. Spinks

University of Wollongong, gspinks@uow.edu.au

Edwin W. Jager

University of Linkoping, edwin@uow.edu.au

Follow this and additional works at: https://ro.uow.edu.au/eispapers

Part of the Engineering Commons, and the Science and Technology Studies Commons

Research Online is the open access institutional repository for the University of Wollongong. For further information contact the UOW Library: research-pubs@uow.edu.au 


\title{
Bottom-up microfabrication process for individually controlled conjugated polymer actuators
}

\begin{abstract}
Handling of soft and fragile sub-millimeter sized samples such as cells and tissues requires new tools that allow delicate manipulation. Conducting polymer actuators show unique characteristics suitable to driving such manipulators, however despite their potential, the current fabrication method of the trilayer structures does not allow constructing advanced micromanipulators operating in air using this technology. Here we show a novel bottom-up microfabrication process for conjugated polymer trilayer actuators using various solid polymer electrolytes. In addition, the process design integrates contact pads, which has been an issue for small scale conducting polymer actuators. The microfabrication process starts with a patterned layer of conjugated polymer, followed by depositing a polymer electrolyte and a second patterning of the second conjugated polymer layer. The process resulted in successful fabrication of individually controllable conducting polymer trilayer actuators comprising polyvinylidenefluoride and poly(vinylidenefluoride-co-hexafluoropropylene) membranes and showed good interfacial adhesion between the different layers in the final device. The polyvinylidenefluoride trilayer actuator showed good actuation capability. The developed bottom-up microfabrication method paves the way for the development of novel micromanipulation tools.
\end{abstract}

\section{Keywords}

controlled, polymer, individually, actuators, process, conjugated, microfabrication, up, bottom

\author{
Disciplines \\ Engineering | Science and Technology Studies
}

\section{Publication Details}

Khaldi, A., Maziz, A., Alici, G., Spinks, G. M. \& Jager, E. W.H. (2016). Bottom-up microfabrication process for individually controlled conjugated polymer actuators. Sensors and Actuators B: Chemical: international journal devoted to research and development of physical and chemical transducers, 230 818-824. 


\section{Bottom-up microfabrication process for 2 individually controlled conjugated 3 polymer actuators}

4 Alexandre Khaldi ${ }^{1}$, Ali Maziz ${ }^{1}$, Gursel Alici ${ }^{2,3}$, Geoffrey M. Spinks ${ }^{2,3,}$ Edwin W.H. Jager, ${ }^{1}$ University, Linköping, Sweden;

2. ARC Centre of Excellence in Electromaterials Science, Intelligent Polymer Research Institute, University of Wollongong, Wollongong, New South Wales 2522, Australia

3. School of Mechanical, Materials and Mechatronic Engineering, University of Wollongong, Wollongong, New South Wales 2522, Australia

\section{ABSTRACT}

Handling of soft and fragile sub-millimeter sized samples such as cells and tissues requires new tools that allow delicate manipulation. Conducting polymer actuators show unique characteristics suitable to driving such manipulators, however despite their potential, the current fabrication method of the trilayer structures does not allow constructing advanced micromanipulators operating in air using this technology. Here we show a novel bottom-up microfabrication process for conjugated polymer trilayer actuators using various solid polymer electrolytes. In addition, the process design integrates contact pads, which has been an issue for small scale conducting polymer actuators. The microfabrication process starts with a patterned layer of conjugated polymer, followed by depositing a polymer electrolyte and a second patterning of the second conjugated polymer layer. The process resulted in successful fabrication of individually controllable conducting polymer trilayer actuators comprising Polyvinylidenefluoride and Poly(vinylidenefluoride-co-hexafluoropropylene) membranes and showed good interfacial adhesion between the different layers in the final device. The Polyvinylidenefluoride trilayer actuator showed good actuation capability. The developed bottom-up microfabrication method paves the way for the development of novel micromanipulation tools.

Highlights

- Bottom up microfabrication of trilayer actuators demonstrated

- Integrating contact pad design for small scale devices

- First working actuator for this fabrication approach

Keywords 
1 Polypyrrole; Actuator; Solid polymer electrolyte; Electroactive polymer, Bottom up

2 microfabrication

3 Abbreviations

4 CP: Conjugated polymers

5 MEMS: Microelectromechanical systems

6 PVDF: Polyvinylidenefluoride

7 PVDF-HFP: Poly(vinylidenefluoride-co-hexafluoropropylene)

8 PEO: Polyethyleneoxyde

9 PC :Propylene carbonate

10 LiTFSI: Lithium bis(trifluoromethane)sulfonimide

11 EMITFSI: 1,3-ethylmethyimidazolium bis(trifluoromethane)sulfonimide

12 AIBN: Azobisisobutyronitrile

13 DMF: Dimethylformamide

PEGDM: Poly(ethylene glycol)dimethacrylate

PEGM: Poly(ethylene glycol)methylethermethacrylate

IPN: Interpenetrating polymer network

PPY: Polypyrrole

SPE: Solid polymer electrolyte

\section{Introduction}

Soft robotics is changing the way we look at robots. A paradigm shift is occurring where hard and rigid robots are intended to be replaced by soft or biomimetic robots to assure safe interaction with humans and its working environment. Soft and compliant actuators are being developed to articulate soft robotic systems. Likewise, soft microrobots and micromanipulation devices need similar soft and compliant actuators. The handling and manipulation of small, sub-millimeter-sized objects under a microscope, micromanipulation, is generally difficult to undertake with manipulation devices made of hard components and requires specialized instruments. Commercial micromanipulators exist, for instance, to probe electrical contacts or manipulate cells or small pieces of tissue. A well-known example of micromanipulation in medicine is the in vitro fertilization technique intracytoplasmic sperm

30 injection. Today, this is realized with a mechanical micromanipulator adjusting rigid pipettes and vacuum suction under optical microscopes to manipulate egg and sperm cells. These methods which require manually handling samples by well-trained operators may lead to 
challenges in reproducibility of the manipulation. Moreover, when the objects are fragile or soft, it becomes even more cumbersome and it may not be possible to handle the objects anymore using vacuum suction. Therefore, new, automated tools are needed to handle fragile, soft objects and tissues and to reduce the use of human manual handling. Throughout the years, different concepts to grasp small objects have been presented in the literature. For instance various MEMS (microelectromechanical systems) grippers have been developed but they are complex to micro-fabricate, brittle, non-compliant and display small deflections [14]. An interesting concept is the use of the jamming effect of granular materials for a gripper which can grab objects with arbitrary shapes [5]. However, this kind of jamming devices are difficult to micro-fabricate and would not allow grabbing soft objects. Rotary micromotors [6, 7], which are difficult to miniaturize, would require a transmission mechanism such as a gear box or push/pull wires to operate a gripper and are non-compliant. Thus, there is a need to develop new micromanipulation tools in cell-biology and medicine as well as to manipulate fragile objects in general.

Active materials that drive such new micromanipulators should be easy to microfabricate, have accurate position control and have compliant mechanical properties to avoid damaging the sample while at the same time producing enough force to displace objects such as cells and tissue. In case of untethered applications, the materials should also have low energy consumption. Conjugated polymers (CPs) are soft smart materials that can be used to design soft actuators with a low foot-print [8-11]. It is a very promising technology since, depending on the materials structures and components, the actuators can operate in liquid, in air and in low pressure environments [12-15]. They present large actuation amplitudes while requiring low voltages in the range of $1-5 \mathrm{~V}$. CPs meet the requirements as actuators for soft, micromanipulation devices as they have been shown to dispense enough force $(\mu \mathrm{N})[16]$ and displacement for micromanipulation and microrobotics. Although the energetic efficiency of $\mathrm{CP}$ actuators is low $(\approx 1 \%)$ in comparison to piezoelectric ceramics actuators which are often used in microscale applications, they output a larger displacement [17-19]. CP actuators can be integrated into flexible electronic arrangements such as microfluidic systems.

CPs can be microfabricated [13, 17], even into complex microrobotic arms [18]. However, all of these systems required operation in a liquid environment.

In 2009, it was demonstrated that using a laser ablation system, it is possible to fabricate micro-sized CP actuators generating a displacement in air [19]. Next, using classical microfabrication methods such as photo-lithography and reactive ion etching, it has been shown that it is possible to collectively microfabricate them in parallel [20]. By decreasing the size of the actuators to the microscale, the fundamental resonance frequency of the one end clamped, the other end free beam (i.e. cantilever beam) increases. Hence, it has been possible to increase the operating frequency of these systems to $1000 \mathrm{~Hz}$, unusually high for an ionic device [21].

However, due to the presence of two conjugated polymer electrodes in the trilayer structure (one on the "backside" and one on the "front"), it has been impossible until now to microfabricate a complex device with at least two individually controlled trilayer 
microactuators operating in air. Moreover, the size of the microbeam makes it difficult to place the clamp that also acts as an electrical contact. Recently, it has been shown that by patterning the gold electrodes on polyvinylidenefluoride (PVDF) commercial porous membranes, it is feasible to fabricate individually addressable trilayer actuators using microfabrication technology. [22] However, this methodology is not suitable for microscale engineering since it uses thick PVDF membrane. In addition, the substrates need to be flipped during the fabrication process in order to pattern both electrodes making the alignment difficult across an optically opaque layer. An alternative solution to the etching of $\mathrm{Au}$ electrodes would be the etching of the conjugated polymer layer in conducting interpenetrating polymer networks. However, this method would still require flipping the film and would result in inhomogeneity in the thickness of materials making it difficult to model. For these reasons, we developed a bottom-up microfabrication process with different polymer electrolytic membranes, designing a layer by layer polymerization method in a classical microfabrication process [23] inspired by the microfabrication methods used to produce an articulated microrobotic arm [18].

The working principle and the structure of the $\mathrm{CP}$ trilayers actuators is shown in figure 1 . The structure is composed of one solid polymer electrolyte (SPE) acting as an ion reservoir between two CP electrodes. When a voltage is applied between the two CP electrodes, one electrode is oxidized while the other is reduced. The oxidation-reduction process is accompanied by an ion motion to compensate the charge created on the polymer backbone and thus keep electroneutrality. The ion movement causes a net volume change in the two electrodes layers leading to the bending of the trilayer device. This bending is fully reversible. For more details on the functioning of CP trilayer actuators, we refer to Zhou et al [24] and Vidal et al [25].
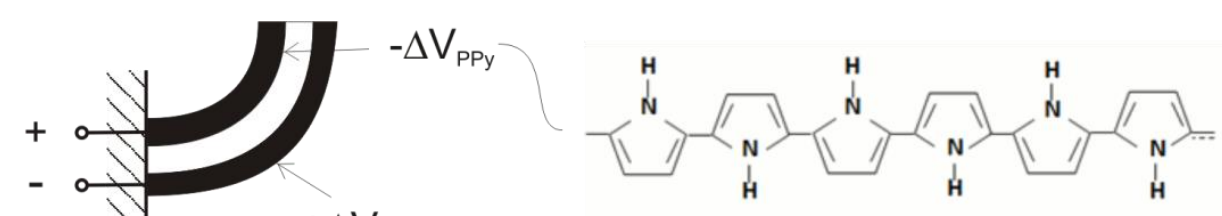

Fig. 1: Principle of conjugated polymer bending actuators based on the oxidation reduction process of PPy. 
1 Typically, for CP actuators, commercial porous PVDF membranes (such as Kynar® or 2 Immobilon-P® membranes) are used as SPE. These commercial membranes are fabricated 3 by phase inversion method. However they are not compatible with a bottom-up microfabrication process as they are too thick and required flipping of the substrate [22]. Gaihre et al have nevertheless shown that using porogens in PVDF solution, it was possible to fabricate thin ionic conductive porous PVDF membrane for actuators fabrication.[26] Other interesting strategies to obtain high conductive membranes can be plasticising PVDF with polymethylmethacrylate in a blend[27], or mixing PVDF-HFP with ionic liquid to produce a highly conductive ionic gel.[28, 29] Indeed the latter has been even used to develop the carbon nanotube based bucky-gel actuators.[29] However, in both of these cases, since the electrolyte is already contained in the membrane and that the patterning of top gold layer and successive electropolymerization required solution processing, the ionic liquid would diffuse out of the membrane and the amount of ionic liquid would be difficult to control preventing reproducibility of the work. An alternative strategy to obtain SPE membranes for CP actuators has been to synthesize interpenetrating polymer networks (IPNs). An IPN is the combination of two or more polymer networks synthesized in juxtaposition, which can be used to combine antagonist properties and functionalities [30]. IPNs result in SPEs that give both a high ionic conductivity as well as good mechanical properties such as high elongation at break [31]. Semi-IPN, which is the combination of one polymer network with one linear polymer, have been used for a top-down approach for the direct synthesis and patterning of $\mathrm{CP}$ actuators on flexible substrates.[30] In this study, we present a newly conceived bottom-up microfabrication process for CP actuators and evaluate various SPE membranes for bottom-up fabrication process.

\section{Materials and Methods}

\subsection{Materials}

Pyrrole was obtained from Sigma-Aldrich, distilled and stored at $-18{ }^{\circ} \mathrm{C}$ prior to usage. Propylene carbonate (PC) and bis(trifluoromethane)sulfonimide lithium salt (LiTFSI), PVDF (powder, $\mathrm{Mn}=534000$ g. $\mathrm{mol}^{-1}$ ), PVDF-HFP (polyvinylidenefluoride-co-hexafluoropropylene, pellets, $\mathrm{Mn}=110000 \mathrm{~g} \cdot \mathrm{mol}^{-1}$ ) and dimethylformamide (DMF) (Sigma-Aldrich) were used as received. $\mathrm{I}_{2}$, KI were acquired from Merck and used as received. Photoresist S1818 and corresponding developer microposit 351 (to dilute) were acquired from Microchem corporation. 4" Si 100 wafer were obtained from semiconductor wafer, Inc. Azobisisobutyronitrile (AIBN) was recrystilized in methanol prior to use, cyclohexanone (Acros, 99.8\%), poly(ethylene glycol) dimethacrylate. (PEGDM, Mn $=750 \mathrm{~g} \mathrm{~mol}^{-1}$, Aldrich), poly(ethylene glycol) methyl ethermethacrylate (PEGM, Mn $=475 \mathrm{~g} \mathrm{~mol}^{-1}$ ) were purchased from sigma-Aldrich. Nitrile butadiene rubber with $44 \mathrm{wt} \%$ acrylonitrile content was obtained from Lanxess.

\subsection{Methods}


2 Microposit S 1818 photoresist was spin-coated at 4000rpm/4000rpm.s ${ }^{-1}$ during 30s using a

3 POLOS spincoater and baked in an oven at $100^{\circ} \mathrm{C}$ during 20 minutes. The photoresist was 4 then exposed for $10 \mathrm{~s}$ using a UV source at $365 \mathrm{~nm},: 10 \mathrm{~mJ} . \mathrm{cm}-2$ intensity, using a Karl Suss MJB-3 mask aligner with a printed photomask from Acreo AB, Norrköping, Sweden. Next the exposed parts were developed using Microposit 351 developer solution.

Gold etchants solution ( $\mathrm{KI} / \mathrm{I}_{2}$ aqueous solution) was prepared by dissolving $4 \mathrm{~g} \mathrm{KI}$ and $2 \mathrm{~g} \mathrm{I}$ in $100 \mathrm{ml} \mathrm{H}_{2} \mathrm{O}$.

The first gold layer is evaporated using an in-house built thermal evaporator at a rate of $5 \AA / s$ at a pressure of 2 mTorr. The second gold layer was deposited using a Vacutec PlasmaSystems sputter system at a pressure of $1.8 \mathrm{mTorr}, 26.0 \mathrm{~cm}^{3} \mathrm{~min}^{-1}$ Ar flow during 5 minutes resulting in a layer of $400 \AA$.

\section{Electropolymerisation}

14 Polypyrrole (PPy) layer is electrodeposited in a two electrode configuration at a constant current of $0.1 \mathrm{~mA} \cdot \mathrm{cm}^{-2}$ using an Ivium compactstat or IviumStat (Ivium Technologies, Eindhoven, The Netherlands) on the patterned gold electrodes from a $0.1 \mathrm{M}$ Pyrrole, $0.1 \mathrm{M}$ LiTFSI and $1 \%$ water in a carbonapropylene solution for 2 hours at $-18{ }^{\circ} \mathrm{C}$. A stainless steel mesh was used as the counter electrode.

Preparation and deposition of solid polymer electrolyte (SPE):

20 PVDF or PVDF-HFP polymers are solubilized in DMF at 200 and $100 \mathrm{~g} . \mathrm{L}^{-1}$ respectively. These solutions were spin-coated on a substrate using a POLOS spincoater, followed by drying at $50{ }^{\circ} \mathrm{C}$ during $30 \mathrm{~min}$ to form a film. In order to obtain pores into the PVDF membrane, an inversion method was used [31]. After spin-coating, and a delay of $5 \mathrm{~s}$, the substrate is immersed into a water bath cooled down to $5^{\circ} \mathrm{C}$.

To prepare polyethyleneoxide-PVDF (PEO-PVDF) semi-interpenetrating polymer network (semi-IPN), first PVDF (1g) was dissolved in DMF (5g) and then PEGM (0.5g), PEGDM $(0.5 \mathrm{~g})$ and $30 \mathrm{mg}$ of AIBN were added to the solution. The mixture was then stirred under nitrogen for $30 \mathrm{~min}$, before spincoating the solution at 500rpm onto the substrate and polymerizing the film for 12 hours at $80^{\circ} \mathrm{C}$ under nitrogen atmosphere.

30 A similar procedure was used to synthesize a PEO-NBR semi IPN. First $1 \mathrm{~g}$ of NBR was dissolved in $5 \mathrm{~g}$ cyclohexanone during 24 hours at room temperature. Thereafter $0,5 \mathrm{~g}$ of PEGM and 0,5g of PEGDM were added to the solution with $30 \mathrm{mg}$ of AIBN. The mixture was then stirred under nitrogen for $30 \mathrm{~min}$, spincoated onto a substrate at 500rpm and polymerized for 12 hours at $80^{\circ} \mathrm{C}$, under nitrogen atmosphere.

The polymer solutions of PVDF, PVDF-HFP and of the precursors of NBR-PEO and PVDFPEO semi-IPNs were spin-coated onto a silicon wafer (with $\mathrm{CP}$ pattern or not) at 500rpm/500rpm. $\mathrm{s}^{-1}$ during $30 \mathrm{~s}$. Then, a curing step respective to each materials followed the deposition. 
2 Profilometry measurments (Dektak 6M) were used to get the thicknesses of the different 3 materials.

4 The actuators were electrically addressed using a compactstat or IviumStat (Ivium 5 technologies) connected using $2 \mathrm{~mm}$ stainless steel crocodile clips on the contact pads, 6 external to the actuators. The displacement was followed by a laser displacement sensor, 7 optoNCDT 1700-50 from Microepsilon (Ortenburg, Germany).

8 The ionic conductivity of the swollen material was measured in a two-electrode system by the 9 complex impedance method using an impedance analyser (Autolab PGSTAT30 with a 10 Frequency Response Analyser module FRA2 from Metrohm). The experiments were 11 performed for an applied peak potential of $5 \mathrm{mV}$ in the frequency range from $0.01 \mathrm{~Hz}$ to 100 $12 \mathrm{MHz}$ at $25^{\circ} \mathrm{C}$. The film was put in a cell with pressure contact stainless steel electrodes.

\section{Results and discussion}

\subsection{General bottom-up microfabrication process}

A classical lithography process was used to pattern two individually controlled actuators. The process flow is shown in figure 2. A gold layer of $500 \AA$ was evaporated onto the silicon substrate. Photoresist was used to pattern the gold layer and the exposed gold surface was wet chemically etched in a $\mathrm{KI} / \mathrm{I}_{2}$ aqueous solution resulting in a first pattern of the bottom electrodes including the contacts pad. Next, the first, bottom polypyrrole (PPy) layer was electropolymerised. A two electrode set-up was used for the electrosynthesis: the patterned $\mathrm{Au}$ electrode was connected to the working electrode lead of the potentiostat and a stainless steel mesh was used as the counter electrode. The reference electrode lead was connected to the counter electrode. The obtained thickness of the PPy layer was $\sim 8 \mu \mathrm{m}$. A SPE was then spin-coated onto the first PPy layer, followed by the appropriate curing step (See section 2.2 for more details about each membrane). The second, top, gold layer was sputtered, resulting in a layer of $400 \AA$. The patterning of the second electrode was realized in the same way as the first. The second PPy layer was then synthesised using the same conditions as the first one. To finish the actuator fabrication, the wafer was immersed in the electrolytic solution of $0.1 \mathrm{M}$ LiTFSI in propylene carbonate a room temperature to swell the SPE and release the device. Finally the actuators were manually cut out using a scalpel. 
Top view

1.

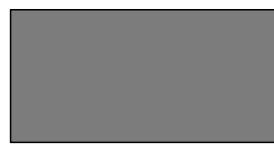

2.

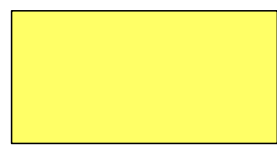

3.

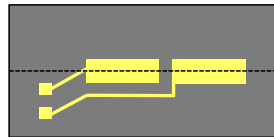

4.

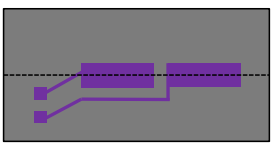

5.

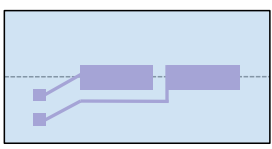

Cross-section

Silicone wafer

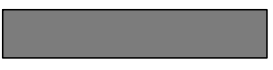

Gold evaporation 200Å

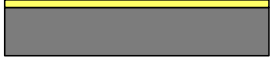

Gold patterning

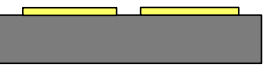

PPy electrodeposition

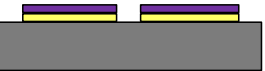

SPE spin-coating
6.

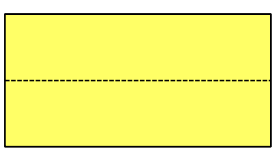

7.

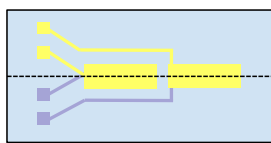

8.

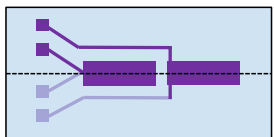

9.

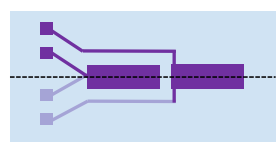

10.

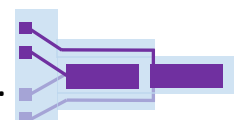

Cross-section
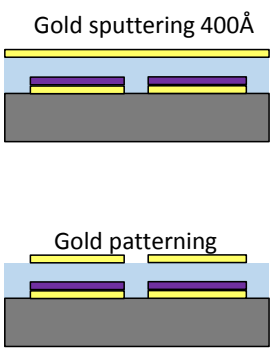

Ppy electrodeposition
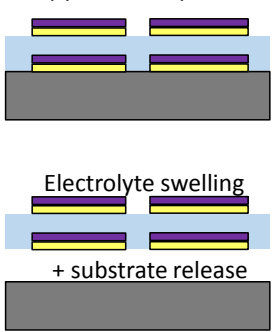

Cut structure

Fig. 2: Process flow for the fabrication of the individually controlled actuators. Step 1 to 4 : deposition of $1^{\text {st }}$ patterned CP electrode. Step 5: deposition of SPE material. Step 6 to 8: deposition of the second patterned CP electrode. Step 9: swelling of the materials by the electrolyte and release of the trilayer structure from the substrate. Step 10: cutting the trilayer structure.

We investigated the feasibility of using this bottom-up microfabrication process with different SPE materials. The swelling ratio of LiTFSI/Propylene carbonate $0.1 \mathrm{M}$ electrolyte, the ionic conductivity, and the advantages and drawbacks of each membrane towards this bottom up microfabrication process are summarized in Table 1.

Table 1: Properties of selected SPE materials

\begin{tabular}{|c|c|c|c|c|c|}
\hline Material & $\begin{array}{c}\text { Swelling } \\
\text { ratio } \\
\text { (wt.\%) }\end{array}$ & $\boldsymbol{\sigma}\left(\mathbf{S . c m}^{-1}\right)$ & Drawback & Advantage & Result \\
\hline PVDF & 55 & $10^{-6}$ & $\begin{array}{c}\text { Low ionic } \\
\text { conductivity }\end{array}$ & $\begin{array}{c}\text { Easy to } \\
\text { process using } \\
\text { spin-coating }\end{array}$ & Final device \\
\hline $\begin{array}{c}\text { PVDF-HFP } \\
\mathbf{1 1 0}\end{array}$ & 46 & $310^{-7}$ & $\begin{array}{c}\text { Low ionic } \\
\text { conductivity }\end{array}$ & $\begin{array}{c}\text { Easy to } \\
\text { process using } \\
\text { spin-coating }\end{array}$ & Final device \\
\hline
\end{tabular}




\begin{tabular}{|c|c|c|c|c|c|}
\hline $\begin{array}{l}\text { PVDF + } \\
\text { pores by } \\
\text { phase } \\
\text { inversion } \\
\text { method }\end{array}$ & -- & -- & $\begin{array}{l}\text { Adhesion to the } \\
\text { substrate, } \\
\text { Shrinking during } \\
\text { the precipitation } \\
\text { of PVDF }\end{array}$ & $\begin{array}{c}\text { Presumably } \\
\text { high ionic } \\
\text { conductivity }\end{array}$ & $\begin{array}{c}\text { SPE } \\
\text { Membrane } \\
\text { deposition } \\
\text { only }\end{array}$ \\
\hline $\begin{array}{c}\text { Phase } \\
\text { separated } \\
\text { PEO-PVDF } \\
\text { semi IPN }\end{array}$ & 575 & $1.510^{-4}$ & $\begin{array}{c}\text { Macroscopic } \\
\text { phase separation }\end{array}$ & $\begin{array}{l}\text { High ionic } \\
\text { conductivity }\end{array}$ & $\begin{array}{c}\text { SPE } \\
\text { Membrane } \\
\text { deposition } \\
\text { only }\end{array}$ \\
\hline $\begin{array}{c}\text { NBR-PEO } \\
\text { semi IPN }\end{array}$ & 350 & $10^{-3}$ & $\begin{array}{l}\text { Swelling with } \\
\text { electrolyte at the } \\
\text { final step lead to } \\
\text { delamination of } \\
\text { multi-layered } \\
\text { device }\end{array}$ & $\begin{array}{c}\text { Ionic } \\
\text { conductivity, } \\
\text { Processability } \\
\text { by spin- } \\
\text { coating }\end{array}$ & $\begin{array}{c}\text { Final device } \\
\text { but electrode } \\
\text { delamination } \\
\text { during } \\
\text { electrolyte } \\
\text { swelling } \\
\text { step }\end{array}$ \\
\hline
\end{tabular}

\subsection{SPE processing and integration in microfabrication}

3 PVDF and PVDF-HFP membranes are used as SPE in actuators for soft microrobotics. These membranes can be easily processed using spin-coating and thermal curing which are common methods employed in microfabrication. As such, we have been able to integrate both SPEs in functional trilayer devices (See Sect. 3.3) However, these membranes exhibit a low ionic conductivity in presence of an electrolyte (respectively $10^{-6}$ and $310^{-7} \mathrm{~S} . c m^{-1}$ for PVDF and PVDF-HFP swollen membranes). Therefore, we also synthesized some new ionic conductive membranes for actuators by a physical method or by chemical engineering.

One way to increase the conductivity is to employ a more porous membrane. We synthesised a porous PVDF membrane by a phase inversion method. In this method, the DMF diffuses out of the PVDF while water penetrates, thus creating a porous structure. At the same time, the PVDF precipitates onto the substrate forming a film. When the PVDF film precipitated on the substrate, a high lateral shrinking was observed often causing a delamination from the underlying substrate, including the first patterned layer. Also, the pores' sizes were relatively large thus preventing the SPE membrane to fully cover the underlying rough PPy layer (see figure 3). These effects prevents the formation of the second patterned electrode in the actuator fabrication process. The porous PVDF SPE made by phase inversion method is an interesting candidate, but requires more development.

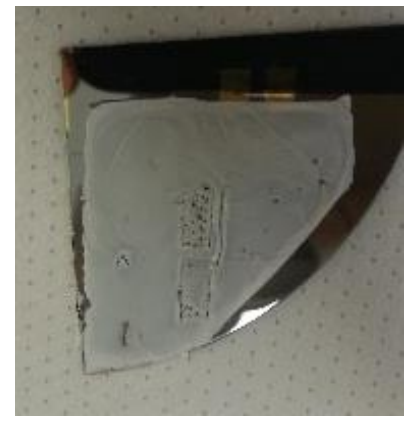


$1 \quad$ Fig 3: Delaminated porous PVDF made by phase inversion method.

2 Next, we synthesized a new semi-IPN based on PVDF physically crosslinked by its 3 crystallites and the PEO network. The material can be processed using spin-coating and cured 4 at $80^{\circ} \mathrm{C}$ in presence of AIBN. The measured ionic conductivity of this this semi-IPN material 5 was high enough $\left(1.510^{-4}{\mathrm{~S} . \mathrm{cm}^{-1}}^{-}\right)$to establish a multi-layer bending actuator. However, macroscopic phase separation in the material occurred, leading to internal stress, causing the multilayer device to curl around itself and delaminate the substrate during the swelling. We believe that this is a promising material and that through more elaborate synthesis study, it may be possible to avoid this phase separation to achieve excellent SPE material properties.

NBR-PEO IPN is often used as the SPE in ionic actuators synthesis, however it cannot be used in this bottom-up microfabrication as the vulcanization of NBR requires a high temperature $\left(180^{\circ} \mathrm{C}\right)$ that would damage the underlying PPy. Therefore, NBR-PEO semi-IPNs were used where only the PEO network is crosslinked at $80^{\circ} \mathrm{C}$, allowing a compatible deposition of this membrane with the bottom-up microfabrication process. Lastly, the PEONBR semi-IPN was then tested with this process. It is an excellent SPE membrane showing excellent properties such as a high ionic conductivity of $10^{-3}{\mathrm{~S} . \mathrm{cm}^{-1}}$ when the material is swollen by LiTFSI/PC or EMITFSI electrolytes. It was possible to go through all the steps of the process and microfabricate the trilayer structure comprising individual electrodes. However, when the material was immersed in the electrolyte, it reached a high weight swelling ratio of $350 \%$ leading to a high volume change of the membrane, causing internal stresses at the gold/PPy-SPE interface causing a delamination of the active parts ( $\mathrm{Au}$ and PPy) from the SPE.

\subsection{Trilayer actuator fabrication}

Using PVDF or PVDF-HFP membranes as the SPE, we were able to successfully process the individually addressable trilayer actuator device using the bottom-up microfabrication process. The final devices are presented in figure 4. We made two different designs each having two individually addressable PPy trilayer actuators of either $5 \times 10 \mathrm{~mm}^{2}$ or $2 \times 4 \mathrm{~mm}^{2}$. The photographs in figure 4 show good resolution of the achieved patterning, thus enabling, in combination with laser ablation techniques, future downscaling to decrease the size of the device to a microscale and the possibility of producing individually controlled microactuators. Moreover, it was possible to add the contact pads for these two actuators. After swelling in the electrolytic solution, the devices were cut out and the actuation characteristics of the built devices were tested. 


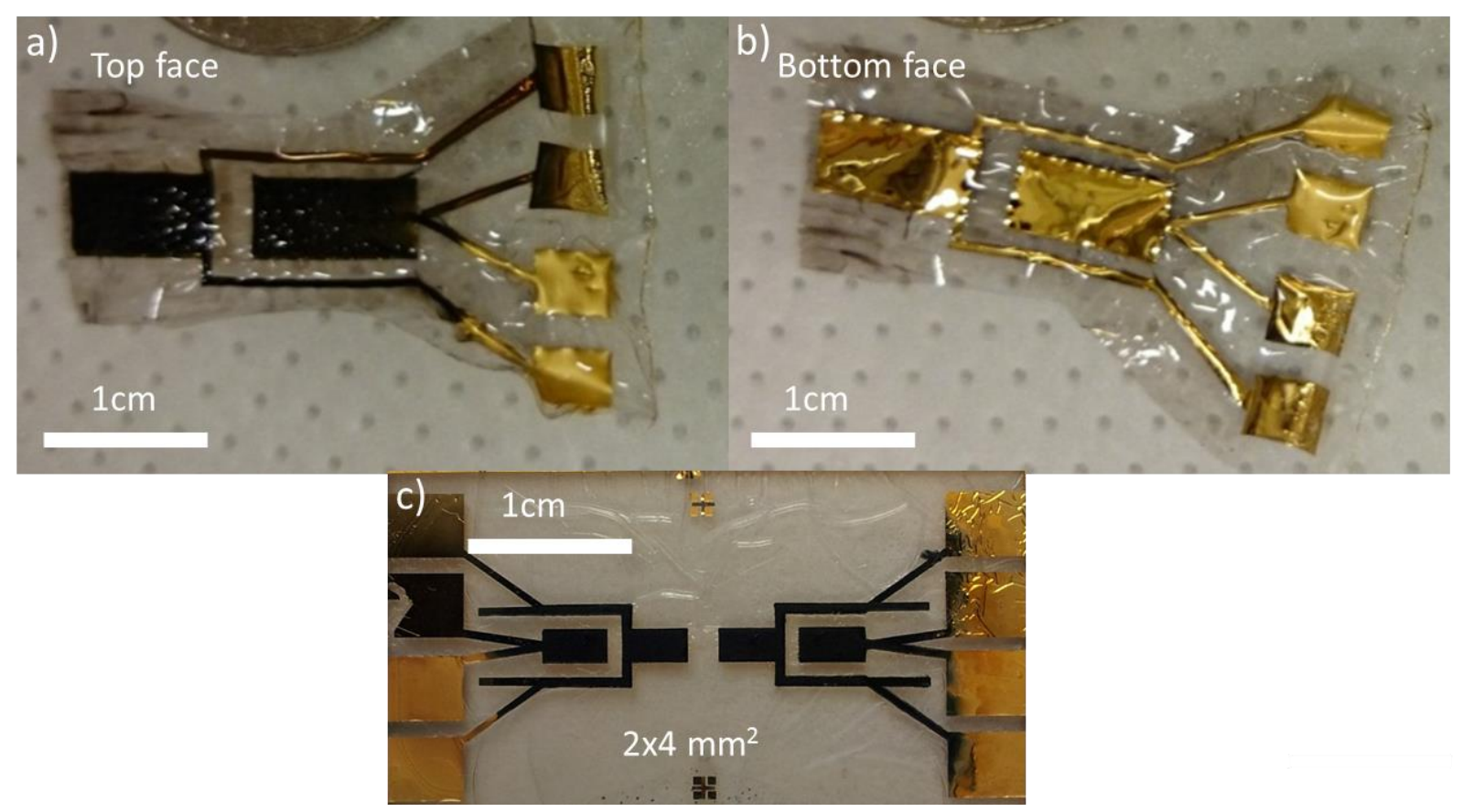

2 Fig 4: Bottom-up fabricated actuator devices (or "fingers") with individually addressable PPy 3 trilayer actuators with $34 \mu \mathrm{m}$ thick PVDF membrane manually cut with a scalpel: a) top face 4 and b) bottom face of a device comprising two $5 \times 10 \mathrm{~mm}^{2}$ sized PPy actuators and c) top face 5 of two devices each comprising two $2 \times 4 \mathrm{~mm}^{2}$ sized PPy actuators. 

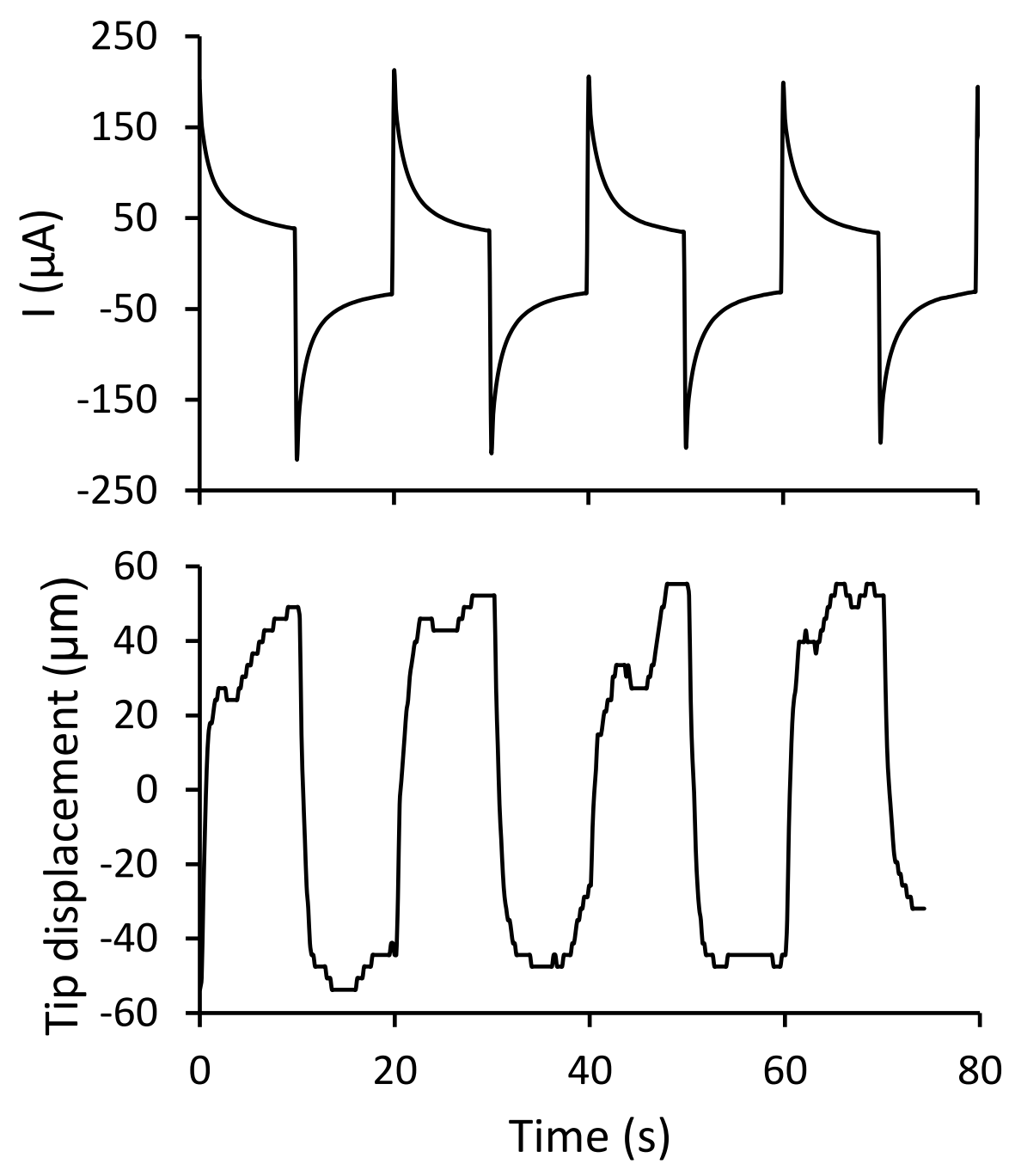

2 Fig 5. Actuation current and displacement of addressing $( \pm 1 \mathrm{~V})$ one of the $5 \times 10 \mathrm{~mm}^{2}$ actuators 3 of a device.

4 Figure 5 shows the actuation current and displacement when addressing one of the $5 \times 10 \mathrm{~mm}^{2}$ 5 actuators of a device. A tip displacement of $100 \mu \mathrm{m}$ was measured at $10 \mathrm{~mm}$ from the contact 6 when $\pm 1 \mathrm{~V}$ was applied at a frequency of $0.05 \mathrm{~Hz}$. From this deflection, the corresponding 7 strain of $10^{-2} \%$ is calculated from the following expression for the strain at any location along 8 the actuator length $\mathrm{L}$;

$9 \varepsilon=2 \frac{h(L-x)^{2}}{L^{4}} y_{\text {tip }}$, for $\mathrm{x}=0, \quad \varepsilon=2 \frac{h}{L^{2}} y_{\text {tip }}$

10 where the variable $\mathrm{x}$ starts from the fixed end of the actuator, $\mathrm{h}$ and $y_{\text {tip }}$ are the total thickness 11 of the actuator and tip displacement of the actuator in the transverse direction, respectively. 12 Eq. 1 assumes that the actuator is modelled as a cantilever beam under a uniformly distributed 13 load [26, 32]. This tip displacement of $100 \mu \mathrm{m}$ is small when compared with the capabilities 14 of such conjugated polymer materials. For instance, Gaihre et al achieved $1.5 \%$ strain 15 applying only a potential of $0.5 \mathrm{~V}$ on thin actuators [26]. We believe this is due to the low 
ionic conductivity of the membrane preventing the ions to be inserted and ejected from the active layer, resulting in as well a limitation of the working frequency. Indeed, if ion motion is restricted, the oxidation-reduction reaction is reduced, thus limiting the volume change and bending of the actuators. To investigate this hypothesis, the actuator device was operated in a $0.1 \mathrm{M} \mathrm{LiTFSI/PC}$ solution, which functions as an external electrolyte and ion source/sink. Inserted in the external electrolyte the devices showed the typical large displacement $(\sim 10$ $\mathrm{mm}$ ) of $\mathrm{CP}$ actuators (indicating a high strain), confirming that there is a problem with the migration of the ions from the electrolytic polymer membrane towards the $\mathrm{CP}$ layers. Unfortunately, our study for better ionically conductive membranes compatible with the bottom-up fabrication process has not yet resulted in finding a SPE membrane for these devices, but both semi-IPN SPEs are very promising candidates that need further investigation. In addition, we are looking for a new way to synthesise flexible and stretchable electrodes. This will simplify the process and enable the use of high ionic conductive membranes with individually controlled CP actuator fabrication.

\section{Conclusion}

A novel fabrication process is reported, showing the feasibility of individually addressable CP actuators operating in air using a bottom-up microfabrication process. The process is applicable to different SPE membranes. Using this process, we have successfully made functional PPy trilayer devices comprising individually addressable actuators. However, the attained displacement of the actuators fabricated with the PVDF as the SPE membrane was smaller in comparison to previously presented CP trilayer actuators [33] due to the low ionic mobility in the employed SPE. Moreover, a promising SPE semi-IPN membrane based on PVDF, polymethacrylate and polyethyleneoxide was synthesized in order to improve ionic conductivity as well as the mechanical properties (such as the Young modulus). This new SPE showed promising homogeneous characteristics in terms of ionic conductivity. An issue with the NBR-PEO semi-IPN SPE is that the gold electrodes used in the process are not stretchable and prevent the use of efficient ionic conductive membrane since the swelling causes the electrodes to delaminate. To overcome this problem and exploit this actuation capacity of the conjugated polymer, our future research will focus on synthesising stretchable electrodes to allow the use of high swollen SPE membranes. We are also currently continuing the work to further scale down the devices and fabricate individually controlled micrometer-sized PPy actuators operating in air. The developed bottom-up microfabrication method presented here paves the way for the development of novel micromanipulation tools.

35 Financial support was received from Linköping University, the EU FP7 Marie Curie action IEF (625923 POLYACT), COST Action MP1003 ESNAM (European Scientific Network for Artificial Muscles) and COST-STSM-MP1003-8971, -13878 \& -16675, the Swedish Research Council (VR-2010-6672 and VR-2014-3079), the Knut \& Alice Wallenberg Stiftelse (LiU-2010-00318, LiU-2012-01361 \& LiU-2014-01752), and Australian Research

40 Council for partial financial assistance through the ARC Centre of Excellence for 
1 Electromaterials Science (CE14010012), and Fellowship Programs (DP110101073). The 2 authors also want to thank Prof Anthony Turner, IFM, Linköping University for his support 3 and LANXESS for graciously providing the NBR.

\section{References}

[1] F. Beyeler, A. Neild, S. Oberti, D. J. Bell, Y. Sun, J. Dual, et al., "Monolithically fabricated microgripper with integrated force sensor for manipulating microobjects and biological cells aligned in an ultrasonic field.", Journal of Microelectromechanical Systems, vol. 16, (2007), pp. 7-15.

[2] S. A. Wilson, R. P. J. Jourdain, Q. Zhang, R. A. Dorey, C. R. Bowen, M. Willander, et al., "New materials for micro-scale sensors and actuators An engineering review", Materials Science \& Engineering R-Reports, vol. 56, (2007), pp. 1-129.

[3] D.S. Chen, C.Y. Yin, R.J. Lai, and J. C. Tsai, "Multiple degrees of freedom electrothermal actuator for a versatile MEMS gripper," in IEEE 22nd International Conference on Micro Electro Mechanical Systems, 2009., Sorrento, 2009, pp. 1035 - 1038.

[4] B.S. Kim, J.S. Park, B.H. Kang, and C. Moon., "Fabrication and property analysis of a MEMS micro-gripper for robotic micro-manipulation.", Robotics and Computer-Integrated Manufacturing, vol. 28, (2012), pp. 50-56.

[5] E. Brown, N. Rodenberg, J. Amend, A. Mozeika, E. Steltz, M. R. Zakin, et al., "Universal robotic gripper based on the jamming of granular material", Proceedings of the National Academy of Sciences of the United States of America, vol. 107, (2010), pp. 18809-18814.

[6] C. H. Ahn, Y. J. Kim, and M. G. Allen, "A planar variable reluctance magnetic micromotor with fully integrated stator and coils", Journal-of-Microelectromechanical-Systems, vol. 2, (1993), pp. 165-173.

[7] B. Wagner, M. Kreutzer, and W. Benecke, "Permanent magnet micromotors on silicon substrates", Journal of Microelectromechanical Systems, vol. 2, (1993), pp. 23-29.

[8] R. H. Baughman, L. W. Shacklette, R. L. Elsenbaumer, E. J. Plichta, and C. Becht, "Micro electromechanical actuators based on conducting polymers," in Molecular Electronics, P. I. Lazarev, Ed., ed Dordrecht: Kluwer Academic Publishers, 1991, pp. 267-289.

[9] Q. Pei and O. Inganäs, "Conjugated polymers as smart materials: bending bipolymer strips," in Smart Materials and Structures, Albuquerque, 1993, pp. 28-39.

[10] E. Smela, "Conjugated polymer actuators for biomedical applications", Advanced Materials, vol. 15, (2003), pp. 481-494.

[11] G. M. Spinks, G. G. Wallace, L. Liu, and D. Zhou, "Conducting polymers electromechanical actuators and strain sensors", Macromolecular Symposia, vol. 192, (2003), pp. 161-169.

[12] R. H. Baughman, "Conducting polymer artificial muscles", Synthetic metals, vol. 78, (1996), pp. 339-353.

[13] E. W. H. Jager, E. Smela, and O. Inganäs, "Microfabricating Conjugated Polymer Actuators", Science, vol. 290, (2000), pp. 1540-1545.

[14] A. Khaldi, C. Plesse, C. Soyer, D. Troadec, F. Vidal, E. Cattan, et al., "Micro-beam actuator based on conducting interpenetrating polymer networks: From patterning process to actuation in open air," in 16th International Solid-State Sensors, Actuators and Microsystems Conference (TRANSDUCERS), 2011 Bejing, China, 2011, pp. 462-465.

[15] A. Punning, K. J. Kim, V. Palmre, F. Vidal, C. Plesse, N. Festin, et al., "Ionic electroactive polymer artificial muscles in space applications", Scientific Reports, vol. 4, (2014), p. 6913.

[16] B. Gaihre, G. Alici, G. M. Spinks, and J. M. Cairney, "Pushing the limits for microactuators based on electroactive polymers", Journal of Microelectromechanical Systems, vol. 21, (2012), pp. 574-585.

[17] E. Smela, O. Inganäs, and I. Lundström, "Controlled folding of micrometer-size structures", Science, vol. 268, (1995), pp. 1735-1738. 
[18] E. W. H. Jager, O. Inganäs, and I. Lundström, "Microrobots for Micrometer-Size Objects in Aqueous Media: Potential Tools for Single Cell Manipulation", Science, vol. 288, (2000), pp. 2335-2338.

[19] G. Alici, V. Devaud, P. Renaud, and G. Spinks, "Conducting polymer microactuators operating in air", Journal of Micromechanics and Microengineering, vol. 19, (2009), p. 025017.

[20] A. Khaldi, C. Plesse, C. Soyer, E. Cattan, F. Vidal, C. Legrand, et al., "Conducting interpenetrating polymer network sized to fabricate microactuators", Appl. Phys. Lett., vol. 98, (2011), p. 164101.

[21] A. Maziz, C. Plesse, C. Soyer, C. Chevrot, D. Teyssié, E. Cattan, et al., "Demonstrating kHz Frequency Actuation for Conducting Polymer Microactuators", Advanced Functional Materials, vol. 24, (2014), pp. 4851-4859.

[22] E. W. H. Jager, N. Masurkar, N. F. Nworah, B. Gaihre, G. Alici, and G. M. Spinks, "Patterning and electrical interfacing of individually controllable conducting polymer microactuators", Sensors and Actuators B: Chemical, vol. 183, (2013), pp. 283-289.

[23] A. Khaldi, A. Maziz, G. Alici, G.M. Spinks, and E. W. H. Jager, "Soft, flexible micromanipulators comprising polypyrrole trilayer microactuators.," in SPIE Smart Structures and Materials+ Nondestructive Evaluation and Health Monitoring. International Society for Optics and Photonics, San Diego, 2015.

[24] D.Z. Zhou, G.M. Spinks, G.G. Wallace, C. Tiyapiboonchaiya, D.R. MacFarlane, M. Forsyth, et al., "Solid state actuators based on polypyrrole and polymer-in-ionic liquid electrolytes", Electrochimica Acta, vol. 48, (2003), pp. 2355-2359.

[25] F. Vidal, C. Plesse, D. Teyssié, and C. Chevrot, "Long-life air working conducting semiIPN/ionic liquid based actuator", Synthetic metals, vol. 142, (2004), pp. 287-291.

[26] B. Gaihre, G. Alici, G. M. Spinks, and J. M. Cairney, "Synthesis and performance evaluation of thin film PPy-PVDF multilayer electroactive polymer actuators", Sensors and Actuators A, vol. 165, (2011), pp. 321-328.

[27] S. Rajendran, O. Mahendran, and T. Mahalingam, "Thermal and ionic conductivity studies of plasticized PMMA/PVdF blend polymer electrolytes", European polymer journal, vol. 38, (2002), pp. 49-55.

[28] J. Fuller, A. C. Breda, and R. T. Carlin, "Ionic liquid- polymer gel electrolytes from hydrophylic and hydrophobic ionic liquids", Journal of Electroanatycal chemistry, vol. 459, (1998), pp. 2934.

[29] Takanori Fukushima, Kinji Asaka, Atsuko Kosaka, and T. Aida, "Fully Plastic Actuator through Layer-by-Layer Casting with Ionic-Liquid-Based Bucky Gel", Angew. Chem. Int. Ed. , vol. 44, (2005), pp. $2410-2413$.

[30] A. Maziz, C. Plesse, C. Soyer, E. Cattan, and F. Vidal, "Top-down Approach for the Direct Synthesis, Patterning, and Operation of Artificial Micromuscles on Flexible Substrates", ACS applied materials \& interfaces., vol. 8, (2015), pp. 1559-1564.

[31] A. Magistris, P. Mustarelli, F. Parazzoli, E. Quartarone, P. Piaggio, and A. Bottino, "Structure, porosity and conductivity of PVdF films for polymer electrolytes. ", Journal of power sources, vol. 97, (2001), pp. 657-660.

[32] G. Alici, "An Effective Modelling Approach to Estimate Nonlinear Bending Behaviour of Cantilever Type Conducting Polymer Actuators", Sensors and Actuators: B. Chemical, vol. 141, (2009), pp. $284-292$.

[33] A. Khaldi, A. Maziz, C. Plesse, C. Soyer, F. Vidal, and E. Cattan, "Synergetic PEDOT degradation during a reactive ion etching process", Sensors and Actuators: B Chemical vol. 229 (2016), pp. 635-645. 